\title{
Rotational Motions Recorded at Hualien During the 2012 Wutai, Taiwan Earthquake
}

\author{
Hung-Chie Chiu ${ }^{1, *}$, Fang-Ju Wu ${ }^{2}$, and Huey-Chu Huang ${ }^{3}$ \\ ${ }^{1}$ Institute of Earth Sciences, Academia Sinica, Taipei, Taiwan \\ ${ }^{2}$ Central Weather Bureau, Taipei, Taiwan \\ ${ }^{3}$ Institute of Seismology, National Chung-Cheng University, Chia-yi, Taiwan
}

Received 25 July 2012, accepted 22 October 2012

\begin{abstract}
It is widely accepted that the effects of rotational motions on seismic waves can be neglected when ground motion is small and might be underestimated in near-fault and/or with extremely large ground motions. However, quantitative validation of these assumptions is still needed. During the 2012 Wutai earthquake in Taiwan $\left(6.4 \mathrm{M}_{\mathrm{L}}\right)$, a set of small rotation ratestrong motion velocity data was recorded at the HWLB (Hualien) seismographic station. This station is $161 \mathrm{~km}$ away from the epicenter and the recorded peak ground acceleration and rotation rate are about $7 \mathrm{~cm} \mathrm{~s}^{-1} \mathrm{~s}^{-1}$ and $0.1 \mathrm{mrad} \mathrm{s} \mathrm{s}^{-1}$, respectively. This data set is useful for evaluating the lower bound of ground motion that can be neglected regarding the effects of rotational motions.

In this study, we apply the algorithm developed by Chiu et al. (2012) and derive the time histories of the rotation angle, the centrifugal acceleration and the gravity effect due to the rotational motions. The results show that all three-component rotation angles are less than $3 \times 10^{-4}$ degrees. The maximum centrifugal acceleration and effect of gravity are about $5 \times 10^{-5}$ and $5 \times 10^{-3} \mathrm{~cm} \mathrm{~s}^{-1} \mathrm{~s}^{-1}$, respectively. Although the amount of induced ground motions due to the rotational motions is small, the effect on the waveforms is significant; the maximum waveform difference before and after rotation-motion correction is about $10 \%$ of the Peak Ground Acceleration. We also found that the effects of rotational motions are always present and are proportional to the ground acceleration except that centrifugal acceleration and the vertical component of gravity effect decrease faster compared to the decrease in ground accelerations.
\end{abstract}

Key words: Wutai earthquake, Rotational motions, Strong motion, Centrifugal acceleration

Citation: Chiu, H. C., F. J. Wu, and H. C. Huang, 2013: Rotational motions recorded at Hualien during the 2012 Wutai, Taiwan earthquake. Terr. Atmos. Ocean. Sci., 24, 31-40, doi: 10.3319/TAO.2012.10.22.01(T)

\section{INTRODUCTION}

Inertial sensors used in an accelerometer for measuring translational ground acceleration can also detect the rotational motion related effects including centrifugal acceleration, gravity (tilt) effects, and effects of rotation frame (coordinate system). These effects are ignored in most analyses of strong-motion data because they are much smaller than that of the corresponding translational motions. However, more and more observations from near-fault and/or extremely large ground motion records indicate that these effects might be underestimated. Therefore, estimating the effect of rotational motions on strong-motion data is impor-

\footnotetext{
* Corresponding author

E-mail: chiu@earth.sinica.edu.tw
}

tant. Chiu et al. (2012) developed a numerical algorithm to calculate these effects using a set of rotation rate-strong motion velocity data and applied it to measurements recorded at HWLB (earthquake epicenter distance $21 \mathrm{~km}$, Magnitude 6.9). They showed that the maximum waveform difference before and after rotation-motion correction is about $10 \%$ of the PGA of the corrected waveform. They also indicated that the induced centrifugal acceleration was much larger than the expected value based on Graizer's estimation (2009). Whether these features can be found in a remote recording is still uncertain.

The February 26, 2012 Wutai inland earthquake registered $\mathrm{M}_{\mathrm{L}}$ 6.4. Both the translational motions and the rotation rate are small but accurately recorded at HWLB by 
a velocity type strong-motion sensor VSE-355G3 and a rotation seismometer R1 at HWLB located $161 \mathrm{~km}$ from the epicenter. In such a case, the incident waves could be a good approximation to a plane wave. In this study we apply the numerical algorithm developed by Chiu et al. (2012) to derive the rotation motion related effects and examine the influence of rotational motions on the translational motions and compare the results with the results for the 2009 M 6.9 earthquake.

\section{DATA}

The data selected in this study were recorded at HWLB during the M 6.4 Wutai earthquake with a focal depth of $26.3 \mathrm{~km}$. The epicenter is located to the southwest of the HWLB and, the epicenter distance is $161 \mathrm{~km}$. More details about the seismographic station at HWLB, including the location, site conditions and instrumentation are given in Chiu et al. (2012).

Our analyses require both the three-component rotation rate and three-component acceleration waveforms in a common timing system as inputs. It is necessary to convert the strong-motion velocity waveforms from VSE-355G3 velocity to acceleration. Since VSE-355G3 seismometers have a flat frequency response from 0.02 to $100 \mathrm{~Hz}$, we did not apply any instrument correction for the velocity waveform here. To covert the velocity waveforms to acceleration, we use numerical differentiation. The sampling interval $(\Delta t)$ of velocity waveform is $0.01 \mathrm{sec}$. For keeping zero-time shift when taking numerical differentiation, we apply a cubic spline interpolation to obtain the velocities at $\mathrm{t}-0.5 \Delta \mathrm{t}$ and $\mathrm{t}$ $+0.5 \Delta \mathrm{t}$ which can be further used in a two-point numerical differentiation to obtain the acceleration at time $t$.

To check this conversion, we compare these derived acceleration waveforms with an independent observation at station HWA019 (Smart24 accelerograph). The comparisons of waveforms between the derived and observed acceleration time histories are shown in Fig. 1a and the magnitude squared coherence of waveform pairs are shown in Fig. 1b. In all the figures presented here, the three-component waveforms and spectra are normalized by their maximum and show the east-west, north-south and up-down components from top to the bottom. For purposes of comparison to Fig. 1, the waveforms and Fourier amplitude spectra of both HWLB and HWA019 are further normalized to the same scale. As shown in Fig. 1a, there is no significant difference between waveforms and the correlation coefficients between these two types of waveforms are $0.9836,0.9771$ and 0.9655 in the EW, NS and UD components. A more detailed comparison is given in Fig. 1b. The coherences of two horizontal components are very close to 1 over a frequency range from 0 to $12 \mathrm{~Hz}$ which implies that two types of waveforms are almost identical. Although the coherence around $5 \sim 7 \mathrm{~Hz}$ is less than 1 for a yet unknown reason, the overall waveform similarity is still high in the vertical component.

The instrument response of $\mathrm{R} 1$ sensor $(30 \mathrm{sec} \sim 50 \mathrm{~Hz}$ version) is corrected by poles and zeros provided by the manufacturer (Eentec). Since the major signal of rotational rate falls in a range from 0.5 to $7 \mathrm{~Hz}$ (Fig. 2b) wherein the $\mathrm{R} 1$ sensor has a flat response, the change in waveforms after instrument correction is small and the correlation coefficients between the waveforms before and after correction are $0.9929,0.9946$ and 0.9961 , respectively for the eastwest, north-south and up-down components. The time shift of the waveform due to the non-zero phase of the R 1 transfer function is also estimated to be less than one sampling interval $(0.01 \mathrm{sec})$.

Because the rotational and the translational sensors have different frequency responses, before using these data to estimate the rotational effects, we apply the same bandpass filter (from 0.5 to $20 \mathrm{~Hz}$ ) to both types of waveforms. The final waveforms after conversion, instrument correction, baseline correction and band-passed filtering are shown in Fig. 2a. The maximum rotation rate of two horizontal components are about $0.1 \mathrm{mrad} \mathrm{s}^{-1}$ while the vertical component is about $5 \times 10^{-2} \mathrm{mrad} \mathrm{s}^{-1}$. The peak ground acceleration is about $7 \mathrm{~cm} \mathrm{~s}^{-1} \mathrm{~s}^{-1}$ in the horizontal component and about $2 \mathrm{~cm} \mathrm{~s}^{-1} \mathrm{~s}^{-1}$ in the vertical component.

\section{METHODS}

The approach used for this study is the same as that used by Chiu et al. (2012). A brief introduction of this method is given in the following discussion.

The explicit form of the equation of motion in the rotation frame is

$\ddot{U}^{R}(t)=A^{R}(t)-\dot{\Theta}^{R}(t) \times \dot{U}^{R}(t)-G^{R}(t)$

In Eq. (1) and the following discussions, the symbols in capital letters represent a vector or a matrix and the superscript " $R$ " of a parameter denotes that the parameter is measured in the rotation frame. In Eq. (1), acceleration $\ddot{U}^{R}$ and $\dot{U}^{R}$ velocity of the ground are two unknowns to be solved; $A^{R}$ and $\dot{\Theta}^{R}$ are the observed acceleration and rotation rate in the rotation frame. The second and the third terms on the right of the equation are effects due to the presence of the rotation motions; the second term is the induced centrifugal acceleration, which is equivalent to the cross product of the rotation rate and velocity vector while the third term $G^{R}$ is the effective gravity on three components. Equation (1) can be rewritten as an ordinary differential equation of $\dot{U}^{R}$

$\frac{d \dot{U}^{R}(t)}{d t}=A^{R}(t)-\dot{\Theta}^{R}(t) \times \dot{U}^{R}(t)-G^{R}(t)$ 
Before calculating $G^{R}$, we need to evaluate Euler's angles $\Psi=(\alpha \beta \gamma)$ which define the transformation between the reference frame and rotational frame. To calculate the Euler's angles as time functions, we need to solve the attitude equation (Lin et al. 2010; Chiu et al. 2012).

$\left(\begin{array}{c}\dot{\alpha} \\ \dot{\beta} \\ \dot{\gamma}\end{array}\right)=\left(\begin{array}{ccc}1 & s_{1} \tan \beta & c_{1} \tan \beta \\ 0 & c_{1} & -s_{1} \\ 0 & s_{1} \sec \beta & c_{1} \sec \beta\end{array}\right)\left(\begin{array}{c}\dot{\theta}_{x} \\ \dot{\theta}_{y} \\ \dot{\theta} z\end{array}\right)$

Equation (3) is another ordinary differential equation of $\alpha, \beta$ and $\gamma$. Solving Eq. (3) gives the time function of $\alpha(\mathrm{t}), \beta(\mathrm{t})$ and $\gamma(\mathrm{t})$. Once we have the Euler's angles, we apply them to calculate $G^{R}$ and to transform the ground motions from rotation frame to that in the reference frame.

(a)

HWLB
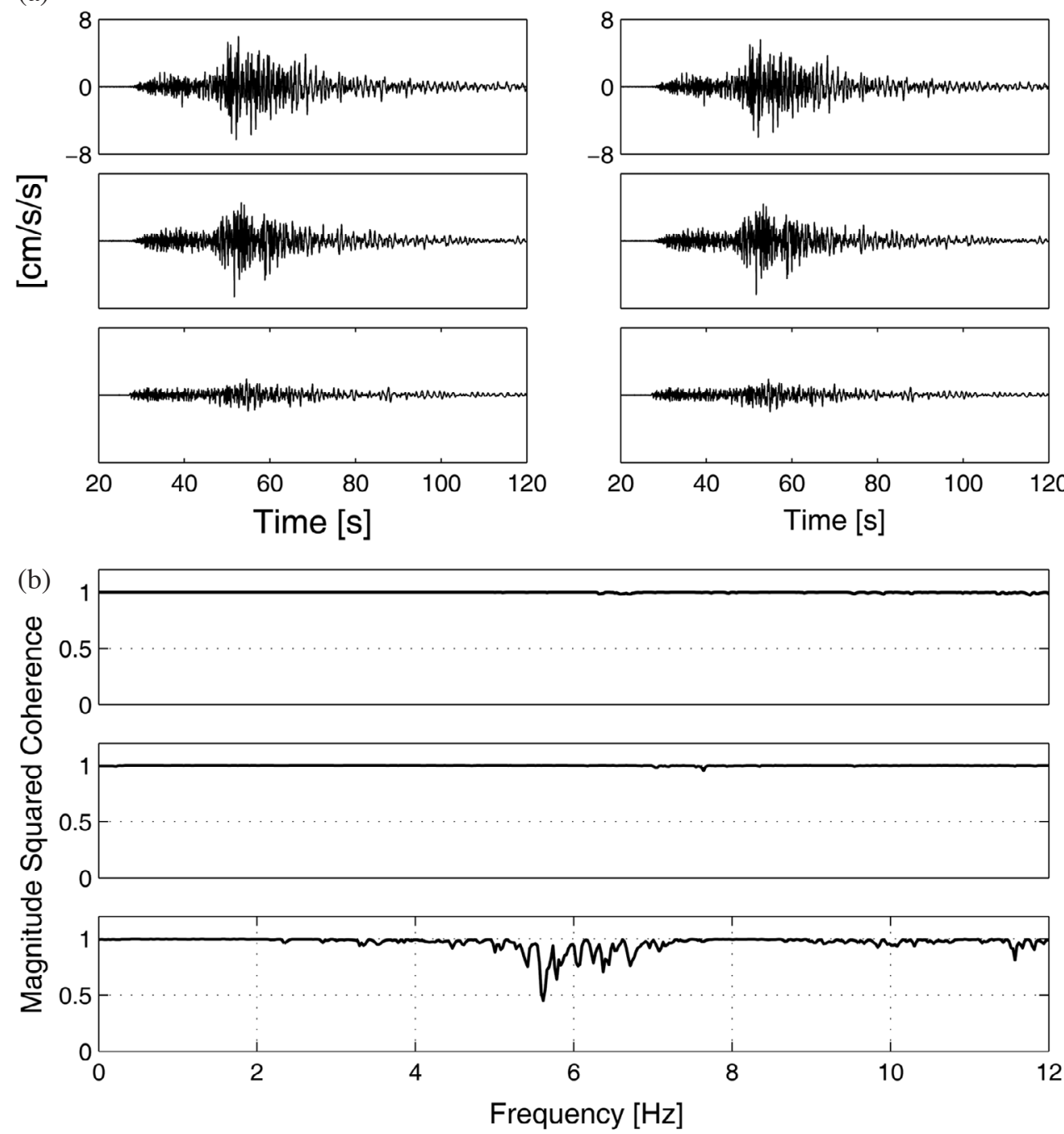

Fig. 1. (a) Side-by-side comparison of waveforms between the derived accelerations (left) and a recorded strong-motion record (right). The derived acceleration is obtained by numerical differentiation of the broadband velocity data recorded at HWLB while the HWA019 is from an independent collocated observation. The magnitude square coherences between these two types of waveforms are shown in (b).

The gravity effect $G^{R}$ in Eq. (2) is the change of gravity before and after the presence of rotation motions, therefore

$$
\begin{aligned}
G^{R} & =G-T_{3} T_{2} T_{1} G \\
& =\left(\begin{array}{l}
0 \\
0 \\
g
\end{array}\right)-\left(\begin{array}{ccc}
c_{3} & -s_{3} & 0 \\
s_{3} & c_{3} & 0 \\
0 & 0 & 1
\end{array}\right)\left(\begin{array}{ccc}
c_{2} & 0 & s_{2} \\
0 & 1 & 0 \\
-s_{2} & 0 & c_{2}
\end{array}\right)\left(\begin{array}{ccc}
1 & 0 & 0 \\
0 & c_{1} & -s_{1} \\
0 & s_{1} & c_{1}
\end{array}\right)\left(\begin{array}{l}
0 \\
0 \\
g
\end{array}\right) \\
& =\left(\begin{array}{c}
-g s_{2} \\
g c_{2} s_{1} \\
-g\left(1-c_{1} c_{2}\right)
\end{array}\right)
\end{aligned}
$$

where $s_{1}=\sin \alpha, c_{1}=\cos \alpha, s_{2}=\sin \beta$, etc.

Substituting (4) and two known variables, $A^{R}$ and $\dot{\Theta}^{R}$, in Eq. (2) gives $\dot{U}^{R}$. The pure translational motion in the rotation frame $\dot{U}^{R}$ can be transformed to the reference frame
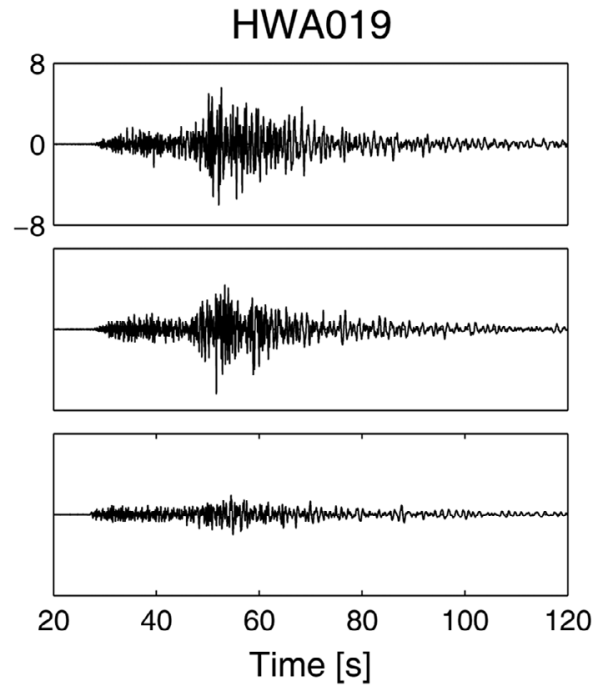

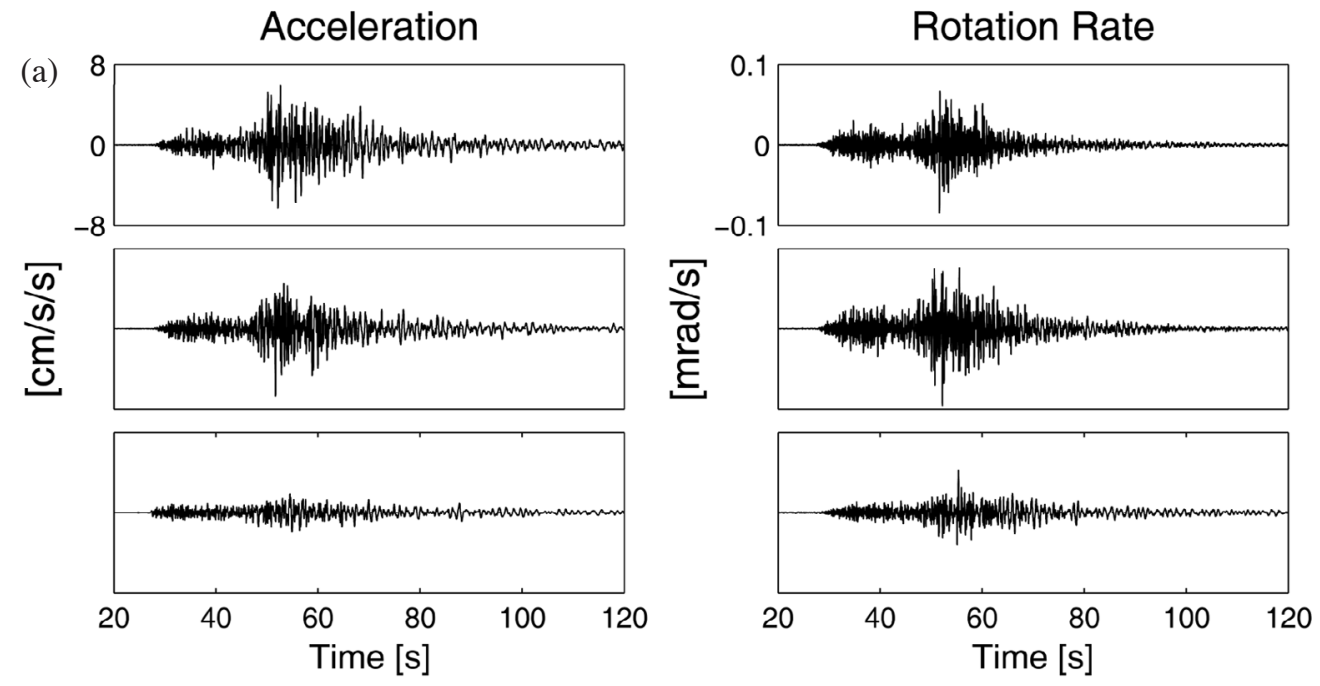

(b)
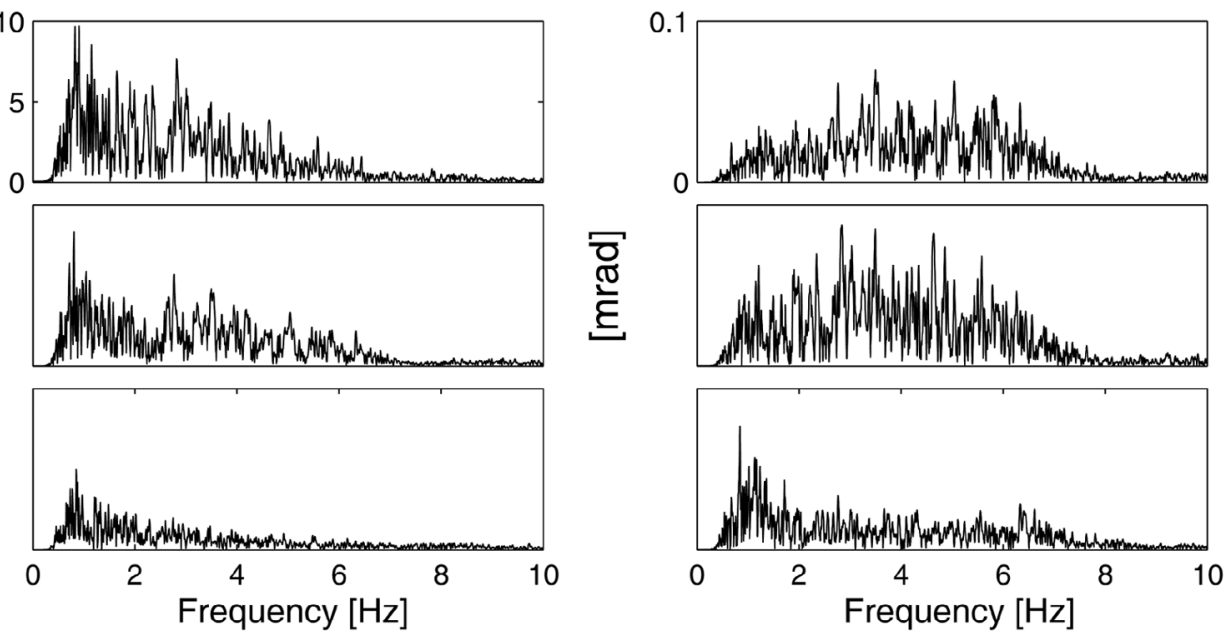

Fig. 2. (a) Three-component rotation rate (left) and ground acceleration (right) used in this study. From top to the bottom are east, north and up directions. The acceleration is derived from the broadband velocity seismogram by numerical differentiation. Their corresponding Fourier amplitude spectra are shown in (b).

using the same transformation matrix as that in Eq. (4) and the result is the translational velocity in the reference frame $\dot{U}$. The translational acceleration and displacement in the reference frame can be derived from $\dot{U}$ by numerically differentiation and integration.

\section{RESULTS AND DISCUSSIONS}

The time histories of Euler's angels $\alpha(\mathrm{t}), \beta(\mathrm{t})$ and $\gamma(\mathrm{t})$ derived from this data set are given in Fig. 3. The maximum Euler's angle shown in Fig. 3 is less than $3 \times 10^{-4}$ degree (Table 1) which indicates that the rotational motions are moving within a very small range.

The three-component centrifugal accelerations, as shown in Fig. 4, have a larger value in the vertical component and show an asymmetric time history. The peak centrifugal acceleration is $4.518 \times 10^{-5} \mathrm{~cm} \mathrm{~s}^{-1} \mathrm{~s}^{-1}$ which is much larger than conventional estimates (e.g., Graizer 2009).
Following Graizer's approach and using an equal length of spring $(20 \mathrm{~cm})$, the induced centrifugal acceleration is less than $2 \times 10^{-7} \mathrm{~cm} \mathrm{~s}^{-1} \mathrm{~s}^{-1}$ as the highest rotation rate and in this case is $9.61 \times 10^{-2} \mathrm{mrad} \mathrm{s}^{-1}$. This estimate is about $0.44 \%$ of our result. In fact, the VSE-355G3 belongs to the masson-spring type sensor which has much shorter length of the spring and which will cause even smaller centrifugal acceleration. Instead of comparing the centrifugal acceleration by assuming that the rotation arm is the same as the length of the spring, we also can estimate the rotation arm using the observed rotation rate and the derived centrifugal acceleration. The estimated lengths of the rotation arm are $48.7 \mathrm{~m}$ for the Wutai earthquake and $38.9 \mathrm{~m}$ for the December 19, 2009 Hualien earthquake. Both cases imply that the center of rotation motion is not at the fixed point of the spring in the seismometer. The R1, VSE-355G3 and HWA019 accelerometer are installed on a $4 \mathrm{~m} \times 3 \mathrm{~m}$ base-isolated concrete pad in the basement of a 2-story building which is $30 \mathrm{~m}$ long 
and $15 \mathrm{~m}$ wide. It seems that the rotation arm is on the same order of size as the size of the building. However, whether the interaction of instrument pad and building can induce such rotational motion requires further investigation.

The estimated effects of gravity using Eq. (4) are shown in Fig. 5. The time histories of the two horizontal components are similar to the ground-motion waveform and both have similar peak values of about $0.005 \mathrm{~cm} \mathrm{~s}^{-1} \mathrm{~s}^{-1}$. It is worth noticing that the effects of gravity in the vertical component $\left(1.47 \times 10^{-8} \mathrm{~cm} \mathrm{~s}^{-1} \mathrm{~s}^{-1}\right)$ are much smaller than those of the two horizontal components and are negative. The negative value is expected because the tilt of instrument always causes a lowered effect in the vertical direction. The smaller effect in vertical component is also expected. For a small ground tilt, the horizontal component is proportional to the tilt angle while the vertical component will be proportional to the square of the tilt angle.

Solving the ordinary differential equation in Eq. (2) gives the three-component velocity waveforms in the rotation frame. Two approaches can be applied to calculate
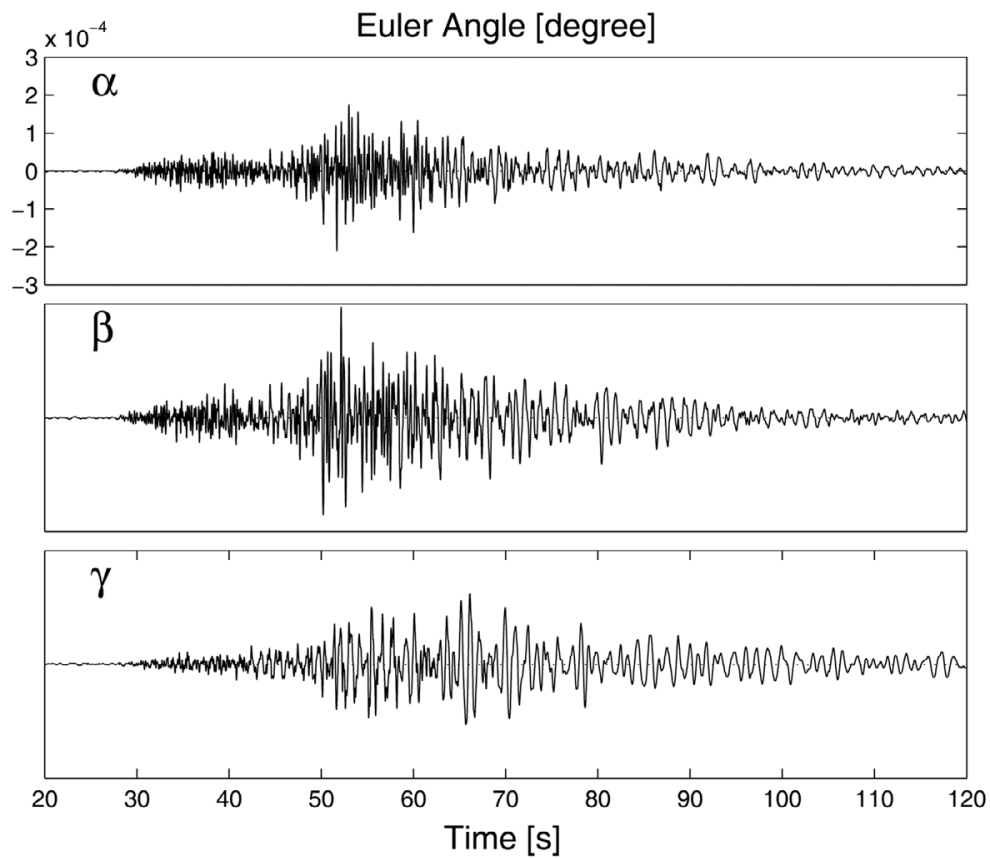

Fig. 3. Three-component Euler's angles in degrees.

Table 1. Three-component peak values of various time histories (column 1) for two earthquakes EQ20091219 (columns $2 \sim 4$ ) and EQ20120226 (columns $5 \sim 7$ ). The corresponding ratios of parameters between two earthquakes are shown in column $8 \sim 10$.

\begin{tabular}{|c|c|c|c|c|c|c|c|c|c|}
\hline & \multicolumn{3}{|c|}{ EQ20091219 } & \multicolumn{3}{|c|}{ EQ20120226 } & \multicolumn{3}{|c|}{ Ratio } \\
\hline & EW & NS & $\mathbf{V}$ & EW & NS & V & EW & NS & $\mathbf{V}$ \\
\hline $\operatorname{rr}\left(\mathrm{mrad} \mathrm{s}^{-1}\right)$ & $2.602 \mathrm{E}+00$ & $1.580 \mathrm{E}+00$ & $7.001 \mathrm{E}-01$ & 8.397E-02 & $9.611 \mathrm{E}-02$ & $5.171 \mathrm{E}-02$ & $3.23 \%$ & $6.08 \%$ & $7.39 \%$ \\
\hline $\mathrm{ac}\left(\mathrm{cm} \mathrm{s}^{-1} \mathrm{~s}^{-1}\right)$ & $1.035 \mathrm{E}+02$ & $1.761 \mathrm{E}+02$ & $4.801 \mathrm{E}+01$ & $6.293 \mathrm{E}+00$ & $6.648 \mathrm{E}+00$ & $1.875 \mathrm{E}+00$ & $6.08 \%$ & $3.78 \%$ & $3.91 \%$ \\
\hline ac_cor $\left(\mathrm{cm} \mathrm{s}^{-1} \mathrm{~s}^{-1}\right)$ & $1.034 \mathrm{E}+02$ & $1.760 \mathrm{E}+02$ & $4.801 \mathrm{E}+01$ & $6.288 \mathrm{E}+00$ & $6.644 \mathrm{E}+00$ & $1.875 \mathrm{E}+00$ & $6.08 \%$ & $3.78 \%$ & $3.91 \%$ \\
\hline vel_cor $\left(\mathrm{cm} \mathrm{s}^{-1}\right)$ & $1.517 \mathrm{E}+01$ & $1.427 \mathrm{E}+01$ & $3.949 \mathrm{E}+00$ & 5.012E-01 & $5.160 \mathrm{E}-01$ & $2.833 \mathrm{E}-01$ & $3.30 \%$ & $3.62 \%$ & $7.17 \%$ \\
\hline dis_cor $(\mathrm{cm})$ & $3.226 \mathrm{E}+00$ & $2.149 \mathrm{E}+00$ & $9.032 \mathrm{E}-01$ & $8.280 \mathrm{E}-02$ & $8.162 \mathrm{E}-02$ & $4.260 \mathrm{E}-02$ & $2.57 \%$ & $3.80 \%$ & $4.72 \%$ \\
\hline EA (degree) & 6.919E-03 & $6.182 \mathrm{E}-03$ & $3.270 \mathrm{E}-03$ & 2.103E-04 & $2.912 \mathrm{E}-04$ & $1.853 \mathrm{E}-04$ & $3.04 \%$ & $4.71 \%$ & $5.67 \%$ \\
\hline $\operatorname{CAC}\left(\mathrm{cm} \mathrm{s}^{-1} \mathrm{~s}^{-1}\right)$ & $4.880 \mathrm{E}-03$ & $7.234 \mathrm{E}-03$ & $2.536 \mathrm{E}-02$ & 1.119E-05 & $1.690 \mathrm{E}-05$ & $4.518 \mathrm{E}-05$ & $0.23 \%$ & $0.23 \%$ & $0.18 \%$ \\
\hline $\mathrm{GE}\left(\mathrm{cm} \mathrm{s}^{-1} \mathrm{~s}^{-1}\right)$ & $1.057 \mathrm{E}-01$ & $1.183 \mathrm{E}-01$ & $7.772 \mathrm{E}-06$ & 4.980E-03 & $3.597 \mathrm{E}-03$ & $1.470 \mathrm{E}-08$ & $4.71 \%$ & $3.04 \%$ & $0.19 \%$ \\
\hline
\end{tabular}

Note: rr: rotation rate; ac: acceleration; ac_cor: corrected Acceleration; vel_cor: corrected Velocity; dis_cor: corrected displacement; EA: Euler's angle; CAC: Centrifugal Acceleration; GE: Gravity Effects. 


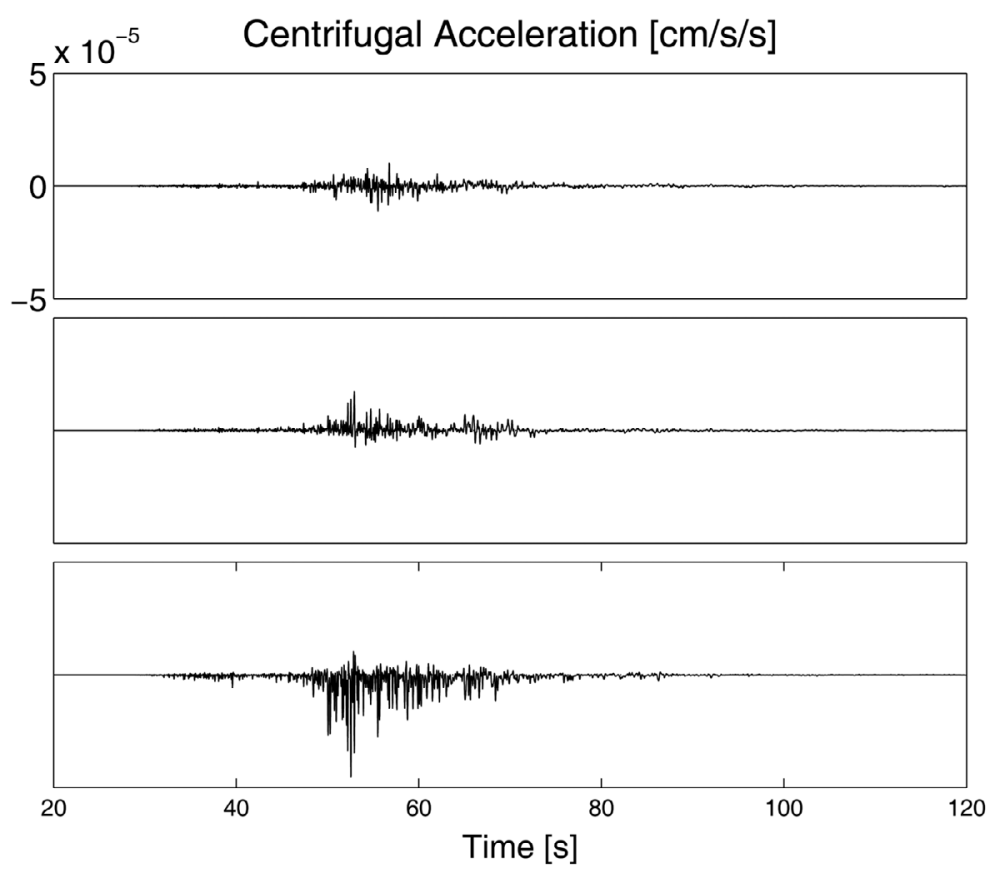

Fig. 4. Three-component centrifugal acceleration due to rotation motions.
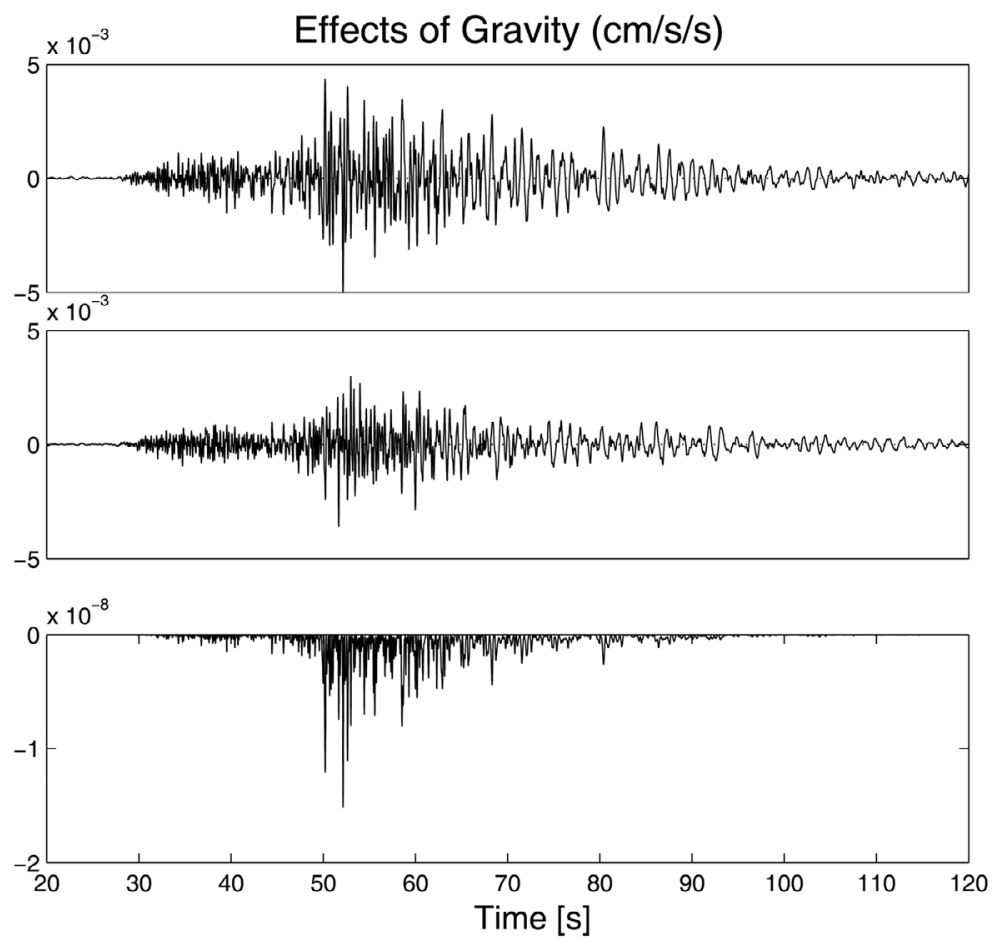

Fig. 5. The gravity effects on the three axes of the rotation frame.

the acceleration waveforms in the rotation frame; one is substituting the velocity in Eq. (1) to obtain $a_{e s t}$, another is applying numerical differentiation on the velocity to give "a." Comparing results of these two approaches provides a chance to check the overall error in numerically solving the ordinary differential Eq. (2) and the numerical differentiation. Comparisons of overlapped waveforms plots are shown in the left of Fig. 6 and the differences of these two waveforms are given on the right of Fig. 6 . As shown in the figure, the difference is about $0.5 \times 10^{-10} \mathrm{~cm} \mathrm{~s}^{-1} \mathrm{~s}^{-1}$ which is 

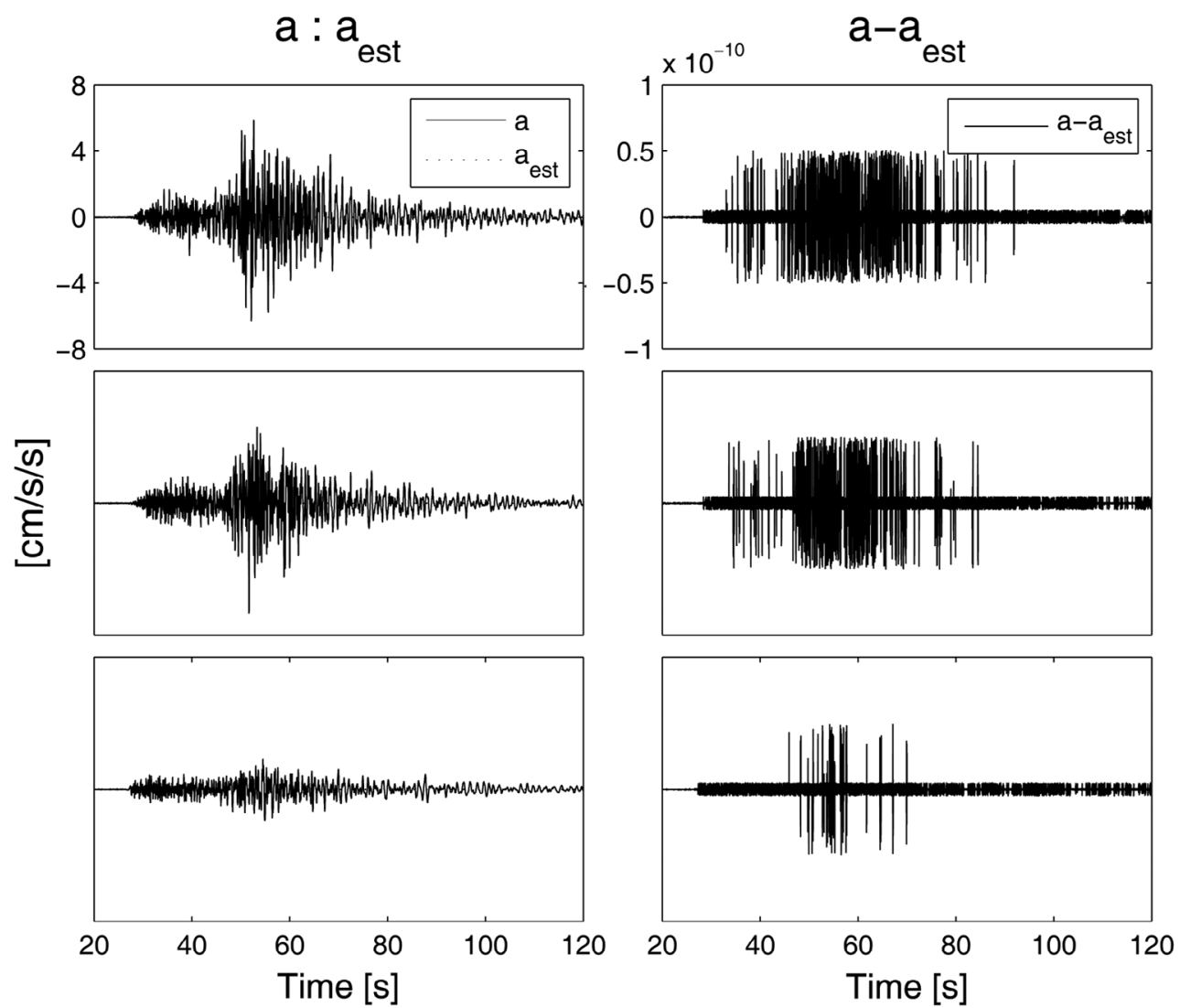

Fig. 6. Comparisons of acceleration waveform between " $a$ " (by numerical differentiation of velocity waveforms) and " $a_{e s t}$ " [by Eq. (2)] are on the left. The differences between " $a$ " and " $a_{\text {est }}$ " are on the right.

much smaller than the peak acceleration of $6.64 \mathrm{~cm} \mathrm{~s}^{-1} \mathrm{~s}^{-1}$.

The ground motions in the reference frame can be considered as corrected waveforms and the effects due to the rotation frame can be examined by comparing the waveforms in both frames. The acceleration waveforms in reference and rotation frame are shown in the first and second columns in Fig. 7 and their difference is given in the third column. The maximum difference is about $0.66,0.73$ and $0.32 \mathrm{~cm} \mathrm{~s}^{-1} \mathrm{~s}^{-1}$ (about 10.4, 9.6 and 11.9\%) for the EW, NS and UD components. This difference has lower frequency content than the original signal and is much larger than the combination of the centrifugal acceleration and gravity effects. Although the ground motion at HWLB in the Wutai earthquake is much smaller (about 3.8\%) than that of the 2009 Hualien earthquake, the percent in waveform difference is almost the same (about $10 \%$ for Hualien earthquake).

The velocity waveforms in reference and rotation frame are shown in the first and second columns in Fig. 8 and their difference is given in the third column. The peak values of these waveform differences are $1.05 \times 10^{-6}, 1.29 \times$ $10^{-6}$ and $1.76 \times 10^{-6} \mathrm{~cm} \mathrm{~s}^{-1}$ for the EW, NS and UD components. Comparing these numbers with the peak values of corrected velocity waveforms, these differences are less than $0.0007 \%$ and can be neglected.
The results of this study for the Wutai earthquake can be compared with that of the 2009 Hualien earthquake where the ground motion was about two orders of magnitude larger. The Hualien earthquake had a larger magnitude (M 6.9) and the epicenter was near HWLB (epicenter distance about $19 \mathrm{~km}$ ). A list of peak values and ratios (Wutai to Hualien) for various time histories of these two earthquakes is given in Table 1. The PGA ratios at HWLB between the Wutai and the Hualien earthquakes are $3.78 \sim 6.08 \%$. These ratios are similar to that of maximum Euler's angle (3.04 $\sim 5.67 \%)$, and maximum horizontal gravity effect (3.04 $4.71 \%)$. On the other hand, ratios of the maximum centrifugal acceleration are $0.18 \sim 0.23 \%$ and the maximum vertical component of gravity is $0.18 \%$ and these four ratios are one order of magnitude smaller than all other ratios. The reason that the gravity effect decreases faster in the vertical component is because its value is in proportional to the square of tilt angle. A possible reason for smaller ratios in the centrifugal acceleration is similar to that of the gravity effect. The scalar value of the centrifugal acceleration $\dot{\Theta}^{R} \times \dot{U}^{R}$ can be written as $r\left(\dot{\theta}^{R}\right)^{2}$ if $r$ is the rotation arm. For small $(<1) \dot{\theta}^{R},\left(\dot{\theta}^{R}\right)^{2}$ drops faster than other ratios that are in proportional to $\dot{\theta}^{R}$.

A linear relationship between peak values of rotational 

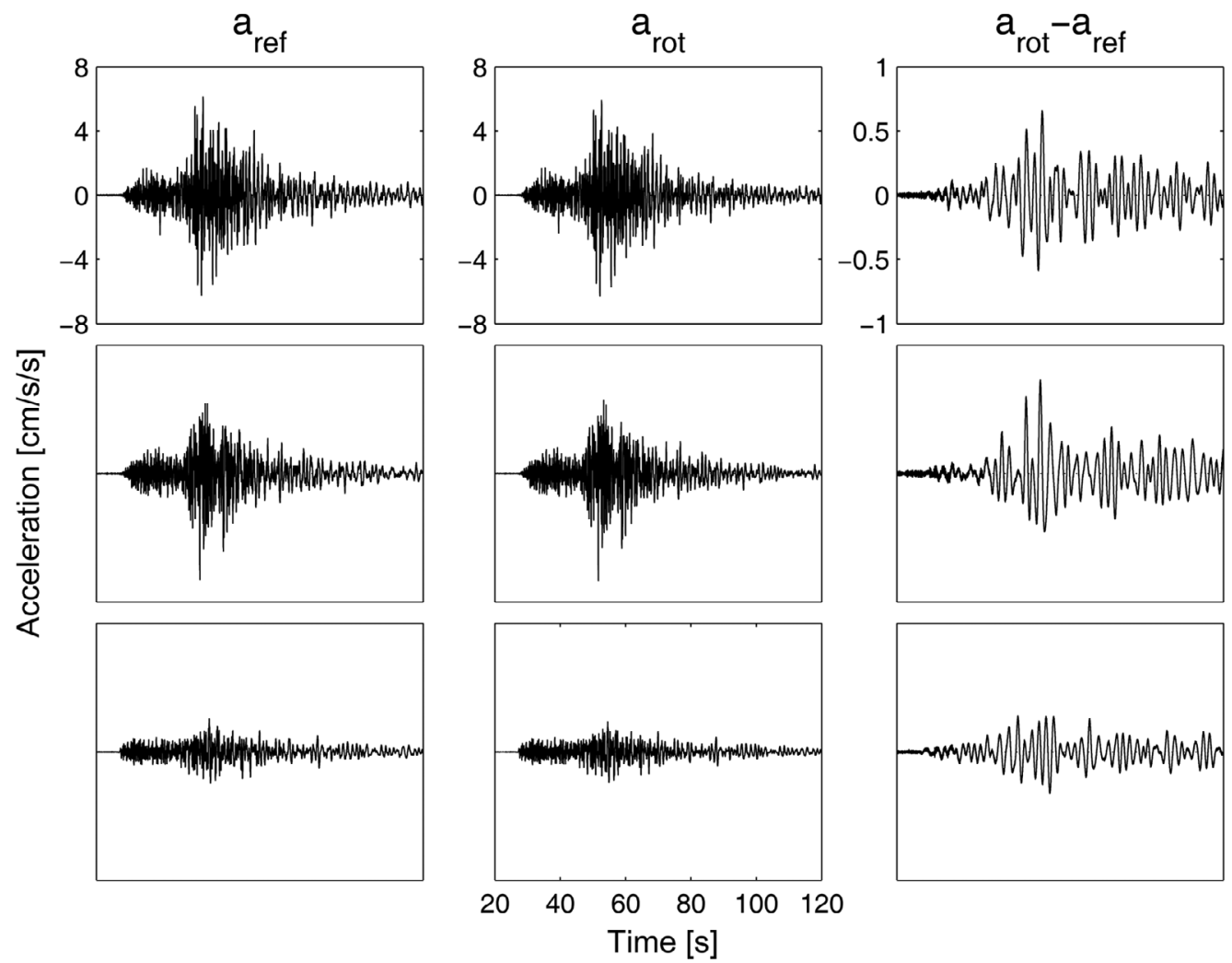

Fig. 7. The three-component accelerations on the reference frame are in the first column while the corresponding components on the rotational frame are in the second column. The differences between these two types of accelerations are shown in the third column.
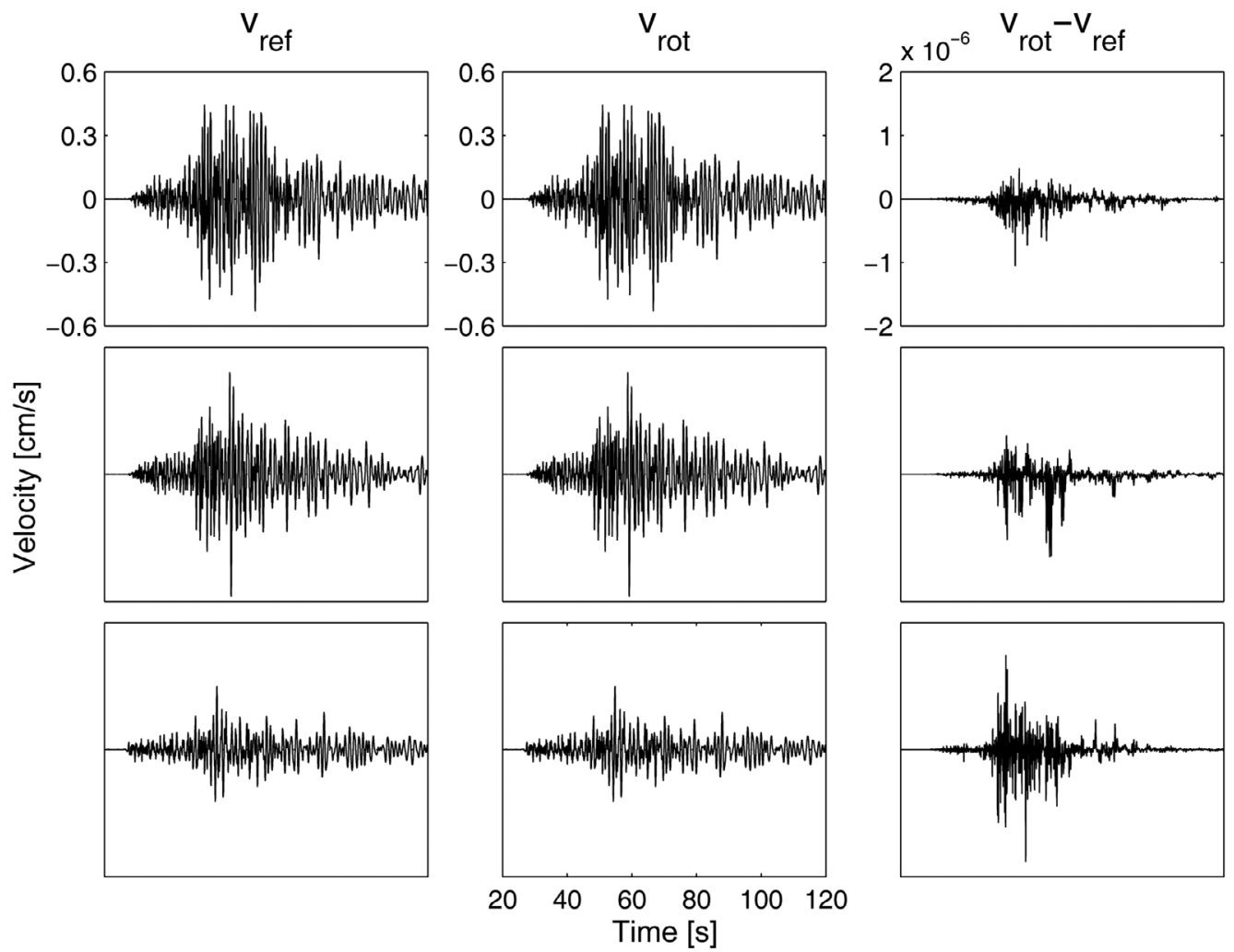

Fig. 8. The three-component velocities on the reference frame are in the first column while the corresponding components on the rotational frame are in the second column. The differences between these two types of accelerations are shown in the third column. 
rate and acceleration (or rotation angle and velocity) has been reported for local earthquakes (Lee et al. 2009; Liu et al. 2009; Takeo 2009; Wu et al. 2009). Determining whether a similar relationship exists for these induced ground motions requires further research. Although only two data points are not adequate to discuss the scaling of effects of rotational motions and translational motions, the trend shows that the effects of rotational motions will grow with the increasing of translational motions. However, more data are needed to establish any relationships, particularly data from areas located between the locations for these two data sets.

Due to the limitation of data, this study only estimates the effects of rotational motions in a limited frequency band from 0.5 to $20 \mathrm{~Hz}$. In selecting the lower bound of the band-pass filter to be $0.5 \mathrm{~Hz}$, we also take the baseline (low-frequency) correction (Chiu 1997, 2012) into account. For the Wutai earthquake, we need a higher lower bound of the band-pass filter. To compare this study with the case of the Hualien earthquake, we use the same upper-cutoff frequency and select a higher lower-cutoff frequency $(0.1 \mathrm{~Hz}$ was selected for the Hualien earthquake) for the band-pass filter to reduce the low-frequency noise. The major signals for both rotational and translational motions are below $7 \mathrm{~Hz}$ (Fig. 2b). The instrument corrections for the high frequencies are not that important for both types of sensors. Without an instrument correction, the R1 data might show a waveform distortion and phase drift at low frequencies (below $1 \mathrm{~Hz}$ ). But the instrument correction for low-frequencies appears to be unneeded for translation data because the flat instrument response can go to zero frequency for most acceleration-type strong-motion sensors. Even for the velocity-type strong-motion sensor such as VSE-355G3, the flat instrument response can go to $0.02 \mathrm{~Hz}$. Therefore, the safe frequency for the lower bound is determined by the rotational-motion sensor and the low-frequency noise in the data.

Common timing for rotation rate and ground motion data is important in our analysis. Therefore, we select the strong-motion velocity waveform rather than use the HWA019 acceleration data. To avoid introducing phase shift into this analysis, the instrument correction and other data manipulations should keep the phase drift to be zero or at a minimum.

\section{CONCLUSIONS}

We apply a numerical algorithm (Chiu et al. 2012) to a six-component ground motion data recorded at HWLB during the 2012 Wutai, Taiwan earthquake to estimate the effects of rotational motions on the strong-motion record. Effects of rotation motions on the translational motions include centrifugal acceleration, gravity effects and the effects of the rotation frame. Since all these analyses are in the time domain, the results of this study provide some detailed fea- tures of these effects. We also compared results with that of the 2009 Hualien earthquake (M 6.9).

The centrifugal acceleration calculated in this study is very small, $4.518 \times 10^{-5} \mathrm{~cm} \mathrm{~s}^{-1} \mathrm{~s}^{-1}$, which is only $0.18 \%$ of that for the Hualien earthquake. The estimated rotation arms in both the cases are on the same order $(48.7 \mathrm{~m}$ for the Wutai earthquake and $37.8 \mathrm{~m}$ for the Hualien earthquake) and these two cases are much larger than the conventional estimate of Graizer (2009).This discrepancy implies that the center of the rotation motion might be not at the fixed point of the pendulum. The cause of this discrepancy needs further study.

The maximum differences between the corrected and uncorrected acceleration waveforms are in the range from 0.32 to $0.73 \mathrm{~cm} \mathrm{~s}^{-1} \mathrm{~s}^{-1}$ (about $9.6 \sim 11.9 \%$ of the corresponding waveforms). This difference has lower frequency content than that of the original signal and is much larger than the combination of the centrifugal acceleration and gravity effects. The percent difference is similar to that of the 2009 Hualien earthquake (about 10\%). The ground the peak ground acceleration of the Wutai earthquake is only $3.8 \%$ of that of the 2009 Hualien earthquake.

Comparisons between the results of the Wutai and Hualien earthquakes show that effects of rotational earthquake still exist in smaller and distant earthquakes. Ratios of peak ground acceleration, maximum Euler's angles, and maximum gravity effects between the Wutai and the Hualien earthquake are on the same order. However, the calculated centrifugal acceleration and the vertical component of gravity effects of the Wutai earthquake is one order of magnitude less than that of the Hualien earthquake. Overall, the effect of rotational motions is still significant in the Wutai earthquake.

Acknowledgements This research was supported by Academia Sinica and the National Science Council, Taiwan, Republic of China (NSC 100-2119-M-001-017). The HWA019 strong-motion data was provided by the Central Weather Bureau, Taiwan. Support from these organizations is gratefully acknowledged. We are also grateful to Dr. Chien-Hsin Chang for the CWB data, an anonymous reviewer, Dr. Wei-An Chao, and Dr. Yi-Ming Wu for their comments and suggestions.

\section{REFERENCES}

Chiu, H. C., 1997: Stable baseline correction of digital strong-motion data. Bull. Seismol. Soc. Am., 87, 932944.

Chiu, H. C., 2012: A compatible baseline correction algorithm for strong-motion data. Terr. Atmos. Ocean. Sci., 23, 171-180, doi: 10.3319/TAO.2011.10.25.01(T). [Link]

Chiu, H. C., F. J. Wu, C. J. Lin, H. C. Huang, and C. C. Liu, 
2012: Effects of rotation motions on strong-motion data. J. Seismol., 16, 829-838, doi: 10.1007/s10950012-9301-z. [Link]

Graizer, V., 2009: Tutorial on measuring rotations using multipendulum systems. Bull. Seismol. Soc. Am., 99, 1064-1072, doi: 10.1785/0120080145. [Link]

Lee, W. H. K., B. S. Huang, C. A. Langston, C. J. Lin, C. C. Liu, T. C. Shin, T. L. Teng, and C. F. Wu, 2009: Review: Progress in rotational ground-motion observations from explosions and local earthquakes in Taiwan. Bull. Seismol. Soc. Am., 99, 958-967, doi: 10.17 85/0120080205. [Link]

Lin, C. J., H. P. Huang, C. C. Liu, and H. C. Chiu, 2010: Application of rotational sensors to correcting rotationinduced effects on accelerometers. Bull. Seismol. Soc.
Am., 100, 585-597, doi: 10.1785/0120090123. [Link]

Liu, C. C., B. S. Huang, W. H. K. Lee, and C. J. Lin, 2009: Observing rotational and translational ground motions at the HGSD station in Taiwan from 2007 to 2008. Bull. Seismol. Soc. Am., 99, 1228-1236, doi: 10.1785/ 0120080156. [Link]

Takeo, M., 2009: Rotational motions observed during an earthquake swarm in April 1998 offshore Ito, Japan. Bull. Seismol. Soc. Am., 99, 1457-1467, doi: 10.1785/ 0120080173. [Link]

Wu, C. F., W. H. K. Lee, and H. C. Huang, 2009: Array deployment to observe rotational and translational ground motions along the Meishan fault, Taiwan: A progress report. Bull. Seismol. Soc. Am., 99, 1468-1474, doi: 10.1785/0120080185. [Link] 